\title{
Effects of nonlinearity of $f(R)$ gravity and perfect fluid in Kaluza-Klein models with spherical compactification
}

\author{
Ezgi Canay $^{1, \mathrm{a}}$, Maxim Eingorn ${ }^{2, \mathrm{~b}}$, Alexander Zhuk $^{3, \mathrm{c}}$ \\ ${ }^{1}$ Department of Physics, Istanbul Technical University, Maslak, 34469 Istanbul, Turkey \\ 2 Department of Mathematics and Physics, North Carolina Central University, Fayetteville st. 1801, Durham, NC 27707, USA \\ ${ }^{3}$ Astronomical Observatory, Odessa National University, Dvoryanskaya st. 2, Odessa 65082, Ukraine
}

Received: 10 March 2020 / Accepted: 24 April 2020 / Published online: 7 May 2020

(C) The Author(s) 2020

\begin{abstract}
We study the effects associated with nonlinearity of $f(R)$ gravity and of the background perfect fluid manifested in the Kaluza-Klein model with spherical compactification. The background space-time is perturbed by a massive gravitating source which is pressureless in the external space but has an arbitrary equation of state $(\mathrm{EoS})$ parameter in the internal space. As characteristics of a nonlinear perfect fluid, the squared speeds of sound are not equal to the background EoS parameters in the external and internal spaces. In this setting, we find exact solutions to the linearized Einstein equations for the perturbed metric coefficients. For nonlinear models with $f^{\prime \prime}\left(R_{0}\right) \neq 0$, we show that these coefficients acquire correction terms in the form of two summed Yukawa potentials and that in the degenerated case, the solutions are reduced to a single Yukawa potential with some "corrupted" prefactor (in front of the exponential function), which, in addition to the standard $1 / r$ term, contains a contribution independent of the three-dimensional distance $r$. In the linear $f^{\prime \prime}(R)=0$ model, we generalize the previous studies to the case of an arbitrary nonlinear perfect fluid. We also investigate the particular case of the nonlinear background perfect fluid with zero speed of sound in the external space and demonstrate that a non-trivial solution exists only in the case of $f^{\prime \prime}\left(R_{0}\right)=0$.
\end{abstract}

\section{Introduction}

Remaining within the framework of the standard fourdimensional General Relativity and the Standard Model of particle physics, it still has not been possible to satisfactorily solve a number of fundamental problems such as the dark

\footnotetext{
a e-mail: ezgicanay@itu.edu.tr

b e-mail: maxim.eingorn@gmail.com

c e-mail: ai.zhuk2@gmail.com (corresponding author)
}

energy and the dark matter problem and neither to unify all fundamental interactions into a single theory. A possible way to settle these problems consists in modifying the Theory of General Relativity and among such attempts, nonlinear $f(R)$ theories [1-6] and theories with extra dimensions [7-11] have gained particular popularity.

It is indeed appealing to study models which combine both approaches, in other words, to consider nonlinear $f(R)$ models in multidimensional space-time. As regards the cosmological aspects, such models were investigated in [1218], where the authors focused mainly on the problems of internal space stabilization, early and late time accelerated expansion of the Universe and dark matter in the form of gravexcitons/radions. To be viable candidates, these models are required to satisfy the gravitational tests carried out in the Solar system, viz., the deflection of light, time delay of radar echoes and perihelion shift. Strictly speaking, the parametrized post-Newtonian (PPN) parameter $\gamma$ obtained for these models must be in concordance with the constraints imposed by such experimental data. For multidimensional models, the astrophysical setting is the most appropriate to study these effects [19-21], as it is also for the General Relativity (see, e.g., [22]). In this setting, the static background metric defined on the product manifold $M=M_{4} \times M_{d}$ (where $M_{4}$ describes external four-dimensional flat spacetime and $M_{d}$ corresponds to the $d$-dimensional internal space) is perturbed by a compact gravitating mass and then, the perturbed metric coefficients are investigated in the weak field limit.

The above mentioned approach with respect to the multidimensional nonlinear $f(R)$ models was applied in [23,24], where the extra dimensions were toroidally compactified and hence, the internal space was not curved. One of the main features of this model, brought about by nonlinearity, is that the metric coefficients receive correction terms in the form of the Yukawa potential. The corresponding Yukawa mass reads 
[23] $m_{\text {scal }}=\left[-(D-1) f^{\prime}\left(R_{0}\right) /\left(2 D f^{\prime \prime}\left(R_{0}\right)\right)+R_{0} / D\right]^{1 / 2}$, where $D$ denotes the number of spatial dimensions, $R_{0}$ denotes the background value of the scalar curvature $\left(R_{0}=0\right.$ in the case of flat background space-time) and the prime denotes differentiation with respect to $R$. Such massive scalar degree of freedom (dubbed scalaron in [25]) is known to be a characteristic feature of the nonlinear models (see, e.g., [2,26-29]). In papers [23,24], a point-like matter source is assigned dust-like equation of state (EoS) in our external space and some arbitrary EoS parameter $\Omega$ in the internal space. The nonzero negative $\Omega$ (i.e., tension in the internal space) is then found to be the necessary condition for the PPN parameter $\gamma$ to satisfy the experimental constraints. The particular value $\Omega=-1 / 2$, which is the black brane/string condition [20,21], presents an interesting case, since gravitating matter sources in the form of black branes/strings do not destroy the stabilization of the internal space $[20,21,30]$.

As indicated previously, in multidimensional nonlinear $f(R)$ models considered in $[23,24]$, both the internal and external background spaces are flat. Namely, background matter is absent. To make the background curved, one needs to introduce a background perfect fluid. The linear multidimensional models with spherical compactification of the internal space were considered in [31-35]. It was shown that the background matter responsible for the internal space curvature has the vacuum-like EoS parameter $\bar{\omega}_{0}=-1$ in the external space and some positive EoS parameter $\bar{\omega}_{1}>0$ in the internal space. It is known [36] that (i) monopole form fields and the Casimir effect can produce such EoS and (ii) this form of matter stabilizes the internal space (see also [31,34]). In linear models with spherical compactification, corrections to the metric coefficients caused by the gravitating mass acquire the form of the Yukawa potential with the Yukawa mass defined by the radius $a$ of the sphere: $m_{\mathrm{rad}} \sim 1 / a$. This scalar degree of freedom (so-called gravexcitons/radions $[36,37]$ ) is a result of the variations in the internal space volume. It was demonstrated in $[31,34]$ that this Yukawa mass and the gravexciton/radion mass are, indeed, exactly equal to each other. For the PPN parameter $\gamma$ to be in agreement with experiments, either the Yukawa mass should be sufficiently large, or for some arbitrary Yukawa mass, the EoS parameter should take on the value $\Omega=-1 / 2$.

What happens now if we consider nonlinear $f(R)$ models with spherical compactification of the internal space? How do scalar degrees of freedom associated with the nonlinearity of the model and with internal space fluctuations compete with each other? This is the main subject of the present paper. We also suppose that the background perfect fluid is nonlinear, i.e., the background parameters $\bar{\omega}_{0}$ and $\bar{\omega}_{1}$ of the EoS in the external and internal spaces are not equal to the squared speed of sound in these spaces. The linear model $f(R)=R$ with spherical compactification and nonlinear background perfect fluid was considered in [38]. Now, we generalize the study for some arbitrary function $f(R)$. First, we obtain the system of linearized equations for perturbations of the metric coefficients. This system has a rather complicated form, but to our surprise, it appears to be exactly solvable for the arbitrary $f(R)$. In the most general case with the condition $f^{\prime \prime}\left(R_{0}\right) \neq 0$, the correction terms to the metric coefficients are found as a combination of two Yukawa potential terms. The associated Yukawa characteristic masses $\mu_{1,2}$, however, are neither equal to $m_{\text {scal }}$ and $m_{\text {rad }}$, nor may be expressed as a simple combination of them. Therefore, the relationship between these masses and $\mu_{1,2}$ is investigated in the relevant limiting cases. For completeness, we also generalize the linear model $f(R)=R+2 \kappa \Lambda_{6}$ previously considered in [31-33] to the case of an arbitrary nonlinear background perfect fluid.

The paper is structured as follows. In Sect. 2, we describe the background model which is perturbed by the massive gravitating source. Here, we derive the system of linearized Einstein equations for the perturbed metric coefficients. This system of equations is solved for nonlinear models with $f^{\prime \prime}\left(R_{0}\right) \neq 0$ in Sect. 3. Then, another particular case, $f^{\prime \prime}\left(R_{0}\right)=0$ with nonlinear perfect fluid is investigated in Sect. 4 . In Sect. 5, we study the case $\omega_{0}=0$ of the nonlinear perfect fluid, viz., zero speed of sound in the external space. In the concluding Sect. 6 we summarize the obtained results. In Appendix we collect the formulas for the perturbations of the Ricci tensor which we use to construct the linearized Einstein equations.

\section{Basic equations}

It is well known (see, e.g., [12,13]) that in the case of $f(R)$ gravity, the Einstein equations take on the form

$$
\begin{aligned}
& f^{\prime}(R) R_{i k}-\frac{1}{2} f(R) g_{i k}-\left[f^{\prime}(R)\right]_{; i ; k}+g_{i k}\left[f^{\prime}(R)\right]_{; m ; n} g^{m n} \\
& \quad=\kappa T_{i k}
\end{aligned}
$$

which is valid for an arbitrary number of space-time dimensions. ${ }^{1}$ In six-dimensional space-time, $\kappa \equiv 2 S_{5} \tilde{G}_{6} / c^{4}$ with the total solid angle $S_{5}=2 \pi^{5 / 2} / \Gamma(5 / 2)=8 \pi^{2} / 3$ and the six-dimensional gravitational constant $\tilde{G}_{6}$. The trace of this equation reads

$$
f^{\prime}(R) R-3 f(R)+5\left[f^{\prime}(R)\right]_{; m ; n} g^{m n}=\kappa T,
$$

\footnotetext{
${ }^{1}$ It is well known that $f(R)$ theories are equivalent to scalar-tensor gravity (see, e.g., [1-3]). Thus, the dynamical equations (e.g., Eq. (1)) can also be reformulated accordingly. In the present paper, however, we do not make use of this equivalence and to compare our findings with the previous results obtained in papers $[23,24]$, proceed within the framework of the original $f(R)$ theory instead.
} 
where $T=g^{m n} T_{m n}$ stands for the trace of the energy-momentum tensor (EMT). From this point on, we fix the number of space-time dimensions to six.

In the background, we consider a factorized six-dimensional metric

$d s^{2}=c^{2} d t^{2}-d x^{2}-d y^{2}-d z^{2}-a^{2}\left(d \xi^{2}+\sin ^{2} \xi d \eta^{2}\right)$,

defined on the product manifold $M=M_{4} \times M_{2}$ where $M_{4}$ is the external/our four-dimensional flat space-time and $M_{2}$ describes the internal two-dimensional space, which is a sphere of radius $a=$ const. For the metric (3), the only nonzero components of the Ricci tensor are $R_{44}^{(0)}=1$, $R_{55}^{(0)}=\sin ^{2} \xi$ and the scalar curvature $R_{0}=-2 / a^{2}$. It is worth mentioning that the minus sign in the latter formula follows from the metric adopted here as well as the sign convention for curvature (as in the book [22]).

We suppose that the metric (3) corresponds to the background matter with the EMT

$$
\left(T_{k}^{i}\right)^{(0)}=\operatorname{diag}\left(\bar{\varepsilon},-\bar{p}_{0},-\bar{p}_{0},-\bar{p}_{0},-\bar{p}_{1},-\bar{p}_{1}\right),
$$

where $\bar{\varepsilon}$ is the background energy density and $\bar{p}_{0}\left(\bar{p}_{1}\right)$ is the background pressure in the external (internal) space. It is thus this matter that curves the background geometry. Again, at the background level, Eqs. (1) and (2) take on the form

$f^{\prime}\left(R_{0}\right) R_{i k}^{(0)}-\frac{1}{2} f\left(R_{0}\right) g_{i k}^{(0)}=\kappa T_{i k}^{(0)}$

and

$$
f^{\prime}\left(R_{0}\right) R_{0}-3 f\left(R_{0}\right)=\kappa T^{(0)},
$$

respectively. From Eq. (5), we obtain the set

$$
\begin{aligned}
-\frac{1}{2} f\left(R_{0}\right) & =\kappa \bar{\varepsilon}, \\
\frac{1}{2} f\left(R_{0}\right) & =\kappa \bar{p}_{0}, \\
f^{\prime}\left(R_{0}\right)+\frac{a^{2}}{2} f\left(R_{0}\right) & =\kappa \bar{p}_{1} a^{2},
\end{aligned}
$$

which implies the following definition for the background EMT:

$T_{i k}^{(0)}= \begin{cases}g_{i k}^{(0)} \bar{\varepsilon} & \text { for } i, k=0, . ., 3 \\ -g_{i k}^{(0)} \bar{p}_{1} & \text { for } i, k=4,5\end{cases}$

These equations demonstrate that the background EoS parameter in the external space $\bar{\omega}_{0} \equiv \bar{p}_{0} / \bar{\varepsilon}=-1$. In order to get the expression for the background EoS parameter $\bar{\omega}_{1} \equiv \bar{p}_{1} / \bar{\varepsilon}$ in the internal space, the form of $f(R)$ should be specified. Plugging in (7)-(10), one may also verify that Eq. (6) is immediately satisfied.
Now, we perturb the background model by a static pointlike massive source smeared over the internal space with mass density $\hat{\rho}(r)=m \delta(\mathbf{r}) / V_{\text {int }}$, where $r=|\mathbf{r}|=$ $\sqrt{x^{2}+y^{2}+z^{2}}$ and the internal space volume $V_{\mathrm{int}}=4 \pi a^{2}$. Since we suppose that this gravitating mass simulates an ordinary astrophysical object, e.g., our Sun, with pressure much less than the energy density, it has the dust-like EoS in the external space. Pressure in the internal space, however, is arbitrary with the EoS parameter $\Omega$. The only nonzero components of the EMT are, then, $\hat{T}_{0}^{0} \approx \hat{\rho} c^{2}$ and $\hat{T}_{k}^{i} \approx-\delta_{k}^{i} \Omega \hat{\rho} c^{2}$ for $i, k=4,5$. Such type of perturbation preserves the blockdiagonal form of the perturbed metric $[31,35]$ :

$$
\begin{aligned}
d s^{2}= & {\left[1+A^{1}(x, y, z)\right] c^{2} d t^{2} } \\
& -\left[1-B^{1}(x, y, z)\right]\left(d x^{2}+d y^{2}+d z^{2}\right) \\
& -\left[a^{2}-G^{1}(x, y, z)\right]\left(d \xi^{2}+\sin ^{2} \xi d \eta^{2}\right),
\end{aligned}
$$

where $A^{1}, B^{1}$ and $G^{1}$ denote the first-order corrections to the metric coefficients. In what follows, we consider the perturbed values $R_{i k}, g_{i k}$ and $T_{i k}$ in the form $A_{i k}=A_{i k}^{(0)}+$ $A_{i k}^{(1)}$ with consecutive terms denoting the background values and first-order perturbations, respectively. By the same reasoning, the perturbed scalar curvature is decomposed as $R=R_{0}+R_{1}$. As for the function $f(R)$, we get

$$
\begin{aligned}
f(R) & =f\left(R_{0}\right)+f^{\prime}\left(R_{0}\right) R_{1}+O\left(R_{1}^{2}\right), \\
f^{\prime}(R) & =f^{\prime}\left(R_{0}\right)+f^{\prime \prime}\left(R_{0}\right) R_{1}+O\left(R_{1}^{2}\right),
\end{aligned}
$$

and Eqs. (1) and (2) accordingly yield

$$
\begin{aligned}
& f^{\prime}\left(R_{0}\right)\left(R_{i k}^{(1)}-\frac{1}{2} g_{i k}^{(0)} R_{1}\right) \\
& \quad-\frac{1}{2} f\left(R_{0}\right) g_{i k}^{(1)}+f^{\prime \prime}\left(R_{0}\right) R_{i k}^{(0)} R_{1} \\
& \quad+\left[g_{i k}^{(0)}\left(R_{1}\right)_{; m ; n} g^{(0) m n}-\left(R_{1}\right)_{i ; k}\right] f^{\prime \prime}\left(R_{0}\right)=\kappa T_{i k}^{(1)} \\
& -2 f^{\prime}\left(R_{0}\right) R_{1}+f^{\prime \prime}\left(R_{0}\right)\left[R_{0} R_{1}+5\left(R_{1}\right)_{; m ; n} g^{(0) m n}\right] \\
& =\kappa T^{(1)} .
\end{aligned}
$$

Here, $T_{i k}^{(1)}$ and $T^{(1)}$ denote the first-order perturbations of the total EMT and its trace, respectively. Again, up to the first order, the EMT of the perturbed background matter reads

$\tilde{T}_{k}^{i} \approx \begin{cases}(\bar{\varepsilon}+\delta \varepsilon) \delta_{k}^{i} & \text { for } i, k=0 ; \\ -\left(\bar{p}_{0}+\delta p_{0}\right) \delta_{k}^{i} \text { for } i, k=1,2,3 ; \equiv\left(T_{k}^{i}\right)^{(0)}+\delta T_{k}^{i}, \\ -\left(\bar{p}_{1}+\delta p_{1}\right) \delta_{k}^{i} \text { for } i, k=4,5\end{cases}$

indicating

$$
\left(T_{k}^{i}\right)^{(1)}=\delta T_{k}^{i}+\hat{T}_{k}^{i}
$$

Let us also assume that

$\delta p_{0}=\omega_{0} \delta \varepsilon, \quad \delta p_{1}=\omega_{1} \delta \varepsilon$, 
where $\omega_{0}$ and $\omega_{1}$ are constant parameters, which are, generally speaking, not connected with the background quantities: $\omega_{0} \neq \bar{\omega}_{0}$ and $\omega_{1} \neq \bar{\omega}_{1}$. Background matter is thus considered to be some nonlinear perfect fluid and the ratio $\delta p / \delta \varepsilon$, referred to as the squared speed of sound [39], is not equal to the background EoS parameters in the internal and external spaces.

In this setting, we now turn to the Einstein equations (14) and (15), taking into account the expressions for the perturbed Ricci tensor components presented in Appendix. Starting with Eq. (14), from the 00-component we obtain

$\frac{f^{\prime}\left(R_{0}\right)}{2}\left(\Delta_{3} A^{1}-R_{1}\right)-\left(\Delta_{3} R_{1}\right) f^{\prime \prime}\left(R_{0}\right)=\kappa\left(\delta \varepsilon+\hat{\rho} c^{2}\right)$,

where we have also used Eq. (7). The 11-, 22- and 33-components yield

$$
\begin{aligned}
& \frac{f^{\prime}\left(R_{0}\right)}{2}\left[\Delta_{3} B^{1}+\frac{\partial^{2}}{\left(\partial x^{i}\right)^{2}}\left(-A^{1}+B^{1}+2 \frac{G^{1}}{a^{2}}\right)+R_{1}\right] \\
& \quad-\frac{1}{2} f\left(R_{0}\right) B^{1}+\left[\Delta_{3} R_{1}-\frac{\partial^{2} R_{1}}{\left(\partial x^{i}\right)^{2}}\right] f^{\prime \prime}\left(R_{0}\right) \\
& =\kappa\left(-\bar{p}_{0} B^{1}+\delta p_{0}\right), \quad i=1,2,3,
\end{aligned}
$$

and using Eq. (9), the components 44 and 55 take on the form

$$
\begin{aligned}
& \frac{f^{\prime}\left(R_{0}\right)}{2}\left(\Delta_{3} \frac{2 G^{1}}{a^{2}}+2 R_{1}\right)+f^{\prime \prime}\left(R_{0}\right) \frac{2 R_{1}}{a^{2}} \\
& \quad+2\left(\Delta_{3} R_{1}\right) f^{\prime \prime}\left(R_{0}\right) \\
& =-f^{\prime}\left(R_{0}\right) \frac{2 G^{1}}{a^{4}}+2 \kappa\left(\delta p_{1}+\Omega \hat{\rho} c^{2}\right) .
\end{aligned}
$$

The mixed 12-, 13- and 23-components read

$$
\begin{aligned}
& \frac{1}{2} f^{\prime}\left(R_{0}\right)\left(-A^{1}+B^{1}+2 \frac{G^{1}}{a^{2}}\right)_{x y}-f^{\prime \prime}\left(R_{0}\right) \frac{\partial^{2} R_{1}}{\partial x \partial y}=0, \\
& \frac{1}{2} f^{\prime}\left(R_{0}\right)\left(-A^{1}+B^{1}+2 \frac{G^{1}}{a^{2}}\right)_{x z}-f^{\prime \prime}\left(R_{0}\right) \frac{\partial^{2} R_{1}}{\partial x \partial z}=0, \\
& \frac{1}{2} f^{\prime}\left(R_{0}\right)\left(-A^{1}+B^{1}+2 \frac{G^{1}}{a^{2}}\right)_{y z}-f^{\prime \prime}\left(R_{0}\right) \frac{\partial^{2} R_{1}}{\partial y \partial z}=0,
\end{aligned}
$$

and in fact, translate into the relation

$$
\frac{1}{2} f^{\prime}\left(R_{0}\right)\left(-A^{1}+B^{1}+2 \frac{G^{1}}{a^{2}}\right)-f^{\prime \prime}\left(R_{0}\right) R_{1}=0,
$$

once we employ the boundary condition that the metric coefficients $A^{1}, B^{1}$ and $G^{1}$, as well as the scalar curvature $R_{1}$, should tend to zero in the limit $r \rightarrow \infty$. We note here that in the linear model with $f^{\prime \prime}\left(R_{0}\right)=0,(25)$ is reduced to its counterpart obtained in papers [31,32].

Using this relation and Eq. (8), we re-express (20) as

$$
\frac{f^{\prime}\left(R_{0}\right)}{2}\left(\Delta_{3} B^{1}+R_{1}\right)+\left(\Delta_{3} R_{1}\right) f^{\prime \prime}\left(R_{0}\right)=\kappa \delta p_{0} .
$$

Combining Eqs. (19), (21), (26) and again, employing the relation (25), we obtain

$$
\begin{aligned}
& 5 f^{\prime \prime}\left(R_{0}\right)\left(\Delta_{3} R_{1}\right)+2 f^{\prime}\left(R_{0}\right) R_{1}+\frac{2}{a^{2}} f^{\prime \prime}\left(R_{0}\right) R_{1} \\
& =-f^{\prime}\left(R_{0}\right) \frac{2 G^{1}}{a^{4}}+\kappa\left[-\delta \varepsilon+\delta p_{0}+2 \delta p_{1}-\hat{\rho} c^{2}(1-2 \Omega)\right] .
\end{aligned}
$$

On the other hand, the trace equation (15) reads

$$
\begin{aligned}
& -2 f^{\prime}\left(R_{0}\right) R_{1}+f^{\prime \prime}\left(R_{0}\right)\left(R_{0} R_{1}-5 \Delta_{3} R_{1}\right) \\
& =\kappa\left[\delta \varepsilon-3 \delta p_{0}-2 \delta p_{1}+\hat{\rho} c^{2}(1-2 \Omega)\right],
\end{aligned}
$$

and added to (27), reveals the relation between $G^{1}$ and $\delta p_{0}$ :

$\delta p_{0}=-\frac{f^{\prime}\left(R_{0}\right)}{\kappa a^{4}} G^{1}$.

Before going further, we make two remarks. First, in the case $f^{\prime \prime}\left(R_{0}\right)=0, f^{\prime}\left(R_{0}\right)=1$ (corresponding also to the linear model $f(R)=R+2 \kappa \Lambda_{6}$ ) and for linear background perfect fluid characterized by $\omega_{0}=\bar{\omega}_{0}, \omega_{1}=\bar{\omega}_{1}$, Eqs. (19), (21), (26) and (28) are reduced to the system of equations (22)(24) investigated earlier in [32]. Therefore, in what follows, we demand $f^{\prime \prime}\left(R_{0}\right) \neq 0$ except for the two particular cases considered in Sects. 4 and 5.2. Second, given the form of the above equations, we should also demand that $f^{\prime}\left(R_{0}\right) \neq 0$. In fact, even the tighter constraint of $f^{\prime}\left(R_{0}\right)>0$ must be imposed so that the graviton is not a ghost $[2,40]$.

Now, we have a system of Eqs. (19), (21) and (26) as well as relations (25) and (29). From Eq. (29) and definitions (18), we get

$\delta \varepsilon=-\frac{f^{\prime}\left(R_{0}\right)}{\kappa a^{4}} \frac{1}{\omega_{0}} G^{1}, \delta p_{1}=-\frac{f^{\prime}\left(R_{0}\right)}{\kappa a^{4}} \frac{\omega_{1}}{\omega_{0}} G^{1}, \omega_{0} \neq 0$,

which, when substituted into (19), (21) and (26) together with the expression for $\delta p_{0}$ from (29), yield

$$
\begin{aligned}
& \frac{f^{\prime}\left(R_{0}\right)}{2} \Delta_{3} B^{1}+\frac{f^{\prime}\left(R_{0}\right)}{2} R_{1}+f^{\prime \prime}\left(R_{0}\right) \Delta_{3} R_{1}+\frac{f^{\prime}\left(R_{0}\right)}{a^{4}} G^{1}=0 \\
& \frac{f^{\prime}\left(R_{0}\right)}{2} \Delta_{3} A^{1}-\frac{f^{\prime}\left(R_{0}\right)}{2} R_{1}-f^{\prime \prime}\left(R_{0}\right) \Delta_{3} R_{1}+\frac{f^{\prime}\left(R_{0}\right)}{a^{4}} \frac{1}{\omega_{0}} G^{1} \\
& =\kappa \hat{\rho} c^{2}, \\
& \frac{f^{\prime}\left(R_{0}\right)}{2 a^{2}} \Delta_{3} G^{1}+\frac{f^{\prime}\left(R_{0}\right)}{2} R_{1}+\frac{f^{\prime \prime}\left(R_{0}\right)}{a^{2}} R_{1}+f^{\prime \prime}\left(R_{0}\right) \Delta_{3} R_{1} \\
& \quad+\frac{f^{\prime}\left(R_{0}\right)}{a^{4}}\left(1+\frac{\omega_{1}}{\omega_{0}}\right) G^{1}=\kappa \Omega \hat{\rho} c^{2} .
\end{aligned}
$$

In the next steps, we proceed to solve the system (31)-(33) together with the relation (25) for the functions $A^{1}, B^{1}, G^{1}$ and $R_{1}$. 


\section{Generic model: $f^{\prime \prime}\left(R_{0}\right) \neq 0, \omega_{0} \neq 0$}

Throughout this section, we study the case of $f^{\prime \prime}\left(R_{0}\right) \neq 0$ (where the linear model with respect to the function $f(R)$ is excluded) for arbitrary values of the EoS parameters $\omega_{0}$ and $\omega_{1}$. In general, these parameters are not equal to the background values $\bar{\omega}_{0}$ and $\bar{\omega}_{1}$, hence, we assume a nonlinear perfect fluid. Additionally, in agreement with Eq. (30), the equations to be solved are valid only for $\omega_{0} \neq 0$.

In attempt to solve the set (31)-(33) and (25) for the functions $A^{1}, B^{1}, G^{1}$ and $R_{1}$, we make use of the Fourier transform

$f(x, y, z)=(2 \pi)^{-3 / 2} \int_{\mathbb{R}^{3}} d \mathbf{k} e^{i \mathbf{k r}} \tilde{f}(\mathbf{k})$.

Provided also that the previously introduced delta-shaped matter source is smeared over the internal space, i.e., $\hat{\rho}=$ $m \delta(\mathbf{r}) / V_{\text {int }}$, the resulting equations read

$$
\begin{aligned}
&- \frac{f^{\prime}\left(R_{0}\right)}{2} k^{2} \tilde{B}^{1}+\frac{f^{\prime}\left(R_{0}\right)}{2} \tilde{R}_{1}-f^{\prime \prime}\left(R_{0}\right) k^{2} \tilde{R}_{1}+\frac{f^{\prime}\left(R_{0}\right)}{a^{4}} \tilde{G}^{1}=0, \\
&- \frac{f^{\prime}\left(R_{0}\right)}{2} k^{2} \tilde{A}^{1}-\frac{f^{\prime}\left(R_{0}\right)}{2} \tilde{R}_{1}+f^{\prime \prime}\left(R_{0}\right) k^{2} \tilde{R}_{1}+\frac{f^{\prime}\left(R_{0}\right)}{a^{4}} \frac{1}{\omega_{0}} \tilde{G}^{1} \\
&= \kappa \frac{m \tilde{\delta}(\mathbf{k})}{V_{\text {int }}} c^{2}, \\
&-\frac{f^{\prime}\left(R_{0}\right)}{2 a^{2}} k^{2} \tilde{G}^{1}+\frac{f^{\prime}\left(R_{0}\right)}{2} \tilde{R}_{1}+\frac{f^{\prime \prime}\left(R_{0}\right)}{a^{2}} \tilde{R}_{1}-f^{\prime \prime}\left(R_{0}\right) k^{2} \tilde{R}_{1} \\
&+\frac{f^{\prime}\left(R_{0}\right)}{a^{4}}\left(1+\frac{\omega_{1}}{\omega_{0}}\right) \tilde{G}^{1}=\kappa \Omega \frac{m \tilde{\delta}(\mathbf{k})}{V_{\text {int }}} c^{2}, \\
& \frac{1}{2} f^{\prime}\left(R_{0}\right)\left(-\tilde{A}^{1}+\tilde{B}^{1}+2 \frac{\tilde{G}^{1}}{a^{2}}\right)-f^{\prime \prime}\left(R_{0}\right) \tilde{R}_{1}=0 .
\end{aligned}
$$

Given the relation $\tilde{\delta}(\mathbf{k})=(2 \pi)^{-3 / 2}$, the solutions $\tilde{A}^{1}(k)$ and $\tilde{B}^{1}(k)$ in the Fourier space are

$$
\begin{aligned}
& \tilde{A}^{1}(k)=\frac{\left(\alpha_{1 A} / \alpha_{4}\right) k^{4}+\left(\alpha_{2 A} / \alpha_{4}\right) k^{2}+\left(\alpha_{3} / \alpha_{4}\right)}{k^{2}\left[k^{4}+\left(\alpha_{5} / \alpha_{4}\right) k^{2}+\left(\alpha_{6} / \alpha_{4}\right)\right]}, \\
& \tilde{B}^{1}(k)=\frac{\left(\alpha_{1 B} / \alpha_{4}\right) k^{4}+\left(\alpha_{2 B} / \alpha_{4}\right) k^{2}+\left(\alpha_{3} / \alpha_{4}\right)}{k^{2}\left[k^{4}+\left(\alpha_{5} / \alpha_{4}\right) k^{2}+\left(\alpha_{6} / \alpha_{4}\right)\right]},
\end{aligned}
$$

where we have introduced the set of coefficients

$$
\begin{aligned}
& \alpha_{1 A} \equiv \kappa^{\prime} {\left[4 f^{\prime \prime}\left(R_{0}\right) a^{4} \omega_{0}(2+\Omega)\right], } \\
& \alpha_{1 B} \equiv \kappa^{\prime} {\left[2 f^{\prime \prime}\left(R_{0}\right) a^{4} \omega_{0}(1-2 \Omega)\right], } \\
& \alpha_{2 A} \equiv \kappa^{\prime}\left\{4 f^{\prime \prime}\left(R_{0}\right) a^{2}\left[\Omega\left(2+\omega_{0}\right)-2 \omega_{1}-2 \omega_{0}\right]\right.\left.-f^{\prime}\left(R_{0}\right) a^{4} \omega_{0}(3+2 \Omega)\right\}, \\
&=\kappa^{\prime}\left\{4 f^{\prime \prime}\left(R_{0}\right) a^{2}\left[\Omega\left(1+2 \omega_{0}\right)-\omega_{1}\right]\right. \\
&\left.-f^{\prime}\left(R_{0}\right) a^{4} \omega_{0}(1-2 \Omega)\right\}, \\
& \alpha_{2 B}= \kappa^{\prime}\left\{2 f^{\prime}\left(R_{0}\right) a^{2}\left[\Omega\left(1+\omega_{0}\right)-\omega_{1}\right]+4 f^{\prime \prime}\left(R_{0}\right) \omega_{0}\right\}, \\
& \alpha_{3} \equiv
\end{aligned}
$$

and

$\alpha_{4} \equiv-5 f^{\prime \prime}\left(R_{0}\right) f^{\prime}\left(R_{0}\right) a^{4} \omega_{0}$,

$$
\begin{aligned}
\alpha_{5} \equiv & 2 a^{2} f^{\prime}\left(R_{0}\right)\left[a^{2} f^{\prime}\left(R_{0}\right) \omega_{0}+f^{\prime \prime}\left(R_{0}\right)\left(1+3 \omega_{0}+3 \omega_{1}\right)\right], \\
\alpha_{6} \equiv & f^{\prime}\left(R_{0}\right) \\
& \times\left[2 f^{\prime \prime}\left(R_{0}\right)\left(\omega_{0}-1\right)-a^{2} f^{\prime}\left(R_{0}\right)\left(1+\omega_{0}+2 \omega_{1}\right)\right],
\end{aligned}
$$

together with $\kappa^{\prime} \equiv(2 \pi)^{-3 / 2} V_{\text {int }}^{-1} \kappa m c^{2}$. For $\tilde{G}^{1}(k)$ and $\tilde{R}^{1}(k)$, the solutions have the form

$\tilde{G}^{1}(k)=\frac{\left(\alpha_{1 G} / \alpha_{4}\right) k^{2}+\left(\alpha_{2 G} / \alpha_{4}\right)}{k^{4}+\left(\alpha_{5} / \alpha_{4}\right) k^{2}+\left(\alpha_{6} / \alpha_{4}\right)}$,

$\tilde{R}^{1}(k)=\frac{\left(\alpha_{1 R} / \alpha_{4}\right) k^{2}+\left(\alpha_{2 R} / \alpha_{4}\right)}{k^{4}+\left(\alpha_{5} / \alpha_{4}\right) k^{2}+\left(\alpha_{6} / \alpha_{4}\right)}$.

The coefficients $\alpha_{1 G}$ and $\alpha_{1 R}$ may be expressed in terms of $\alpha_{1 A}$ and $\alpha_{1 B}$ as

$\alpha_{1 G} \equiv\left(\frac{\alpha_{1 A}}{2}-\alpha_{1 B}\right) a^{2}, \quad \alpha_{1 R} \equiv-\frac{f^{\prime}\left(R_{0}\right)}{f^{\prime \prime}\left(R_{0}\right)}\left(\frac{\alpha_{1 B}}{2}\right)$,

and the remaining two coefficients in (39) read

$\alpha_{2 G} \equiv-\kappa^{\prime}\left[2 f^{\prime \prime}\left(R_{0}\right) a^{4} \omega_{0}+f^{\prime}\left(R_{0}\right) a^{6} \omega_{0}(1+2 \Omega)\right]$,

$\alpha_{2 R} \equiv \kappa^{\prime}\left\{2 f^{\prime}\left(R_{0}\right) a^{2}\left[\Omega\left(\omega_{0}-1\right)+\omega_{0}+\omega_{1}\right]\right\}$.

It is worth noting that the conditions $f^{\prime}\left(R_{0}\right) \neq 0$, $f^{\prime \prime}\left(R_{0}\right) \neq 0$, and $\omega_{0} \neq 0$ herein require $\alpha_{4} \neq 0$.

3.1 Different roots: $\mu_{1}, \mu_{2}>0, \mu_{1} \neq \mu_{2}$

In this subsection we consider the case when the polynomial

$k^{4}+\left(\alpha_{5} / \alpha_{4}\right) k^{2}+\left(\alpha_{6} / \alpha_{4}\right)=\left(k^{2}+\mu_{1}^{2}\right)\left(k^{2}+\mu_{2}^{2}\right)$,

has two distinct negative roots $k^{2}=-\mu_{1}^{2}$ and $k^{2}=-\mu_{2}^{2}$. Given the quadratic form of these roots, without loss of generality, we may choose to proceed with the positive values: $\mu_{1}, \mu_{2}>0$.

After some algebra, the functions (36) and (39) can be cast into the form

$$
\begin{aligned}
& \tilde{A}^{1}(k)=\frac{\beta_{1 A}}{k^{2}+\mu_{1}^{2}}+\frac{\beta_{2 A}}{k^{2}+\mu_{2}^{2}}+\frac{\beta_{3}}{k^{2}}, \\
& \tilde{B}^{1}(k)=\frac{\beta_{1 B}}{k^{2}+\mu_{1}^{2}}+\frac{\beta_{2 B}}{k^{2}+\mu_{2}^{2}}+\frac{\beta_{3}}{k^{2}},
\end{aligned}
$$

and

$$
\begin{aligned}
& \tilde{G}^{1}(k)=\frac{\beta_{1 G}}{k^{2}+\mu_{1}^{2}}+\frac{\beta_{2 G}}{k^{2}+\mu_{2}^{2}}, \\
& \tilde{R}^{1}(k)=\frac{\beta_{1 R}}{k^{2}+\mu_{1}^{2}}+\frac{\beta_{2 R}}{k^{2}+\mu_{2}^{2}},
\end{aligned}
$$

with the coefficients

$$
\beta_{1 A(B)} \equiv \frac{\left(\alpha_{1 A(B)} / \alpha_{4}\right) \mu_{1}^{2}-\alpha_{2 A(B)} / \alpha_{4}+\left(\alpha_{3} / \alpha_{4}\right) \mu_{1}^{-2}}{\mu_{1}^{2}-\mu_{2}^{2}},
$$


$\beta_{2 A(B)} \equiv-\frac{\left(\alpha_{1 A(B)} / \alpha_{4}\right) \mu_{2}^{2}-\alpha_{2 A(B)} / \alpha_{4}+\left(\alpha_{3} / \alpha_{4}\right) \mu_{2}^{-2}}{\mu_{1}^{2}-\mu_{2}^{2}}$,

$\beta_{3} \equiv\left(\alpha_{3} / \alpha_{4}\right) \mu_{1}^{-2} \mu_{2}^{-2}$,

$\beta_{1 G(R)} \equiv \frac{\left(\alpha_{1 G(R)} / \alpha_{4}\right) \mu_{1}^{2}-\alpha_{2 G(R)} / \alpha_{4}}{\mu_{1}^{2}-\mu_{2}^{2}}$

$\beta_{2 G(R)} \equiv-\frac{\left(\alpha_{1 G(R)} / \alpha_{4}\right) \mu_{2}^{2}-\alpha_{2 G(R)} / \alpha_{4}}{\mu_{1}^{2}-\mu_{2}^{2}}$.

The terms of the form of $1 / k^{2}$ and $1 /\left(k^{2}+\mu^{2}\right)$ in (43) and (44) correspond to the Newtonian and Yukawa potentials, respectively. Therefore, in the position space we obtain

$$
\begin{aligned}
& A^{1}(r)=\sqrt{\frac{\pi}{2}} \frac{1}{r}\left[\beta_{3}+\beta_{1 A} \exp \left(-\mu_{1} r\right)+\beta_{2 A} \exp \left(-\mu_{2} r\right)\right], \\
& B^{1}(r)=\sqrt{\frac{\pi}{2}} \frac{1}{r}\left[\beta_{3}+\beta_{1 B} \exp \left(-\mu_{1} r\right)+\beta_{2 B} \exp \left(-\mu_{2} r\right)\right], \\
& G^{1}(r)=\sqrt{\frac{\pi}{2}} \frac{1}{r}\left[\beta_{1 G} \exp \left(-\mu_{1} r\right)+\beta_{2 G} \exp \left(-\mu_{2} r\right)\right], \\
& R^{1}(r)=\sqrt{\frac{\pi}{2}} \frac{1}{r}\left[\beta_{1 R} \exp \left(-\mu_{1} r\right)+\beta_{2 R} \exp \left(-\mu_{2} r\right)\right],
\end{aligned}
$$

consistent with the zero boundary condition for $|\mathbf{r}| \rightarrow$ $\infty$. Parameters $\mu_{1,2}^{-1}$ define the characteristic ranges of the Yukawa interaction. For the polynomial (42), we get

$\mu_{1,2}^{2}=\frac{1}{2}\left(\frac{\alpha_{5}}{\alpha_{4}} \pm \frac{\sqrt{\alpha_{5}^{2}-4 \alpha_{4} \alpha_{6}}}{\alpha_{4}}\right)$

which, upon substitution of the explicit expressions in (38), reads

$$
\begin{aligned}
& \mu_{1,2}=\frac{1}{\sqrt{5}} \frac{1}{a}\left[-a^{2} \frac{f^{\prime}\left(R_{0}\right)}{f^{\prime \prime}\left(R_{0}\right)}-\frac{\left(1+3 \omega_{0}+3 \omega_{1}\right)}{\omega_{0}}\right. \\
& \mp \frac{1}{f^{\prime \prime}\left(R_{0}\right) \omega_{0}} \\
& \quad \times\left[a^{4} f^{\prime 2}\left(R_{0}\right) \omega_{0}^{2}+a^{2} f^{\prime}\left(R_{0}\right) f^{\prime \prime}\left(R_{0}\right)\left(-3+\omega_{0}-4 \omega_{1}\right) \omega_{0}\right. \\
& +f^{\prime \prime 2}\left(R_{0}\right) \\
& \left.\left.\quad \times\left(19 \omega_{0}^{2}+2 \omega_{0}\left(-2+9 \omega_{1}\right)+\left(1+3 \omega_{1}\right)^{2}\right)\right]^{1 / 2}\right]^{1 / 2} .
\end{aligned}
$$

In the limiting case $r \rightarrow \infty, A^{1}$ and $B^{1}$ take on the same value

$$
\begin{aligned}
& A^{1}(r \rightarrow \infty)=B^{1}(r \rightarrow \infty)=\sqrt{\frac{\pi}{2}} \frac{1}{r} \beta_{3} \\
& =\sqrt{\frac{\pi}{2}} \frac{1}{r} \frac{\alpha_{3}}{\alpha_{6}} \equiv-\sqrt{\frac{\pi}{2}} \frac{1}{r} \kappa^{\prime} v=-\frac{2}{c^{2}} \frac{1}{4 \pi} \frac{S_{D} \tilde{G}_{\mathcal{D}}}{V_{\mathrm{int}}} \frac{m}{r} \nu,
\end{aligned}
$$

where

$$
v \equiv \frac{2 f^{\prime}\left(R_{0}\right) a^{2}\left[\Omega\left(1+\omega_{0}\right)-\omega_{1}\right]+4 f^{\prime \prime}\left(R_{0}\right) \omega_{0}}{f^{\prime}\left(R_{0}\right)\left[2 f^{\prime \prime}\left(R_{0}\right)\left(\omega_{0}-1\right)-a^{2} f^{\prime}\left(R_{0}\right)\left(1+\omega_{0}+2 \omega_{1}\right)\right]} .
$$

It is well known (see, e.g., [22]) that the metric coefficient $A^{1}$ defines the gravitational potential and from Eq. (52), we see that it tends to the Newtonian potential in the corresponding limit $r \rightarrow \infty$. With this regard, demanding $A^{1}(r \rightarrow \infty)=2 \varphi_{N} / c^{2}$ for $\varphi_{N}=-G_{N} m / r$, we deduce the relation between the multidimensional gravitational constant $\tilde{G}_{\mathcal{D}}$ and the Newtonian gravitational constant $G_{N}$ in the form

$\frac{S_{D} \tilde{G}_{\mathcal{D}}}{V_{\text {int }}} v=4 \pi G_{N}$

For $\omega_{0}=\bar{\omega}_{0}=-1$, the expression (53) for $v$ is significantly simplified to yield

$\frac{S_{D} \tilde{G}_{\mathcal{D}}}{V_{\text {int }}} \frac{1}{f^{\prime}\left(R_{0}\right)}=4 \pi G_{N}$,

which clearly demands the positiveness of $f^{\prime}\left(R_{0}\right)$.

Example: $f(R)=R+\xi R^{2}$

In order to obtain specific estimates, it is necessary to determine the form of the function $f(R)$ and to this end, we consider the popular quadratic model $f(R)=R+\xi R^{2}$. Herein, imposing the condition $f^{\prime \prime}\left(R_{0}\right) \neq 0$ immediately results in $\xi \neq 0$. Requiring $f^{\prime}\left(R_{0}\right)>0$ as well, we obtain the inequality $a^{2}>4 \xi$, which is satisfied for all negative values of $\xi$.

For such choice, the Yukawa parameters $\mu_{1,2}$ (51) read

$$
\begin{aligned}
& \mu_{1,2}=\frac{1}{\sqrt{10}} \frac{1}{a}\left[-\frac{a^{2}}{\xi}-\frac{2\left(1+\omega_{0}+3 \omega_{1}\right)}{\omega_{0}}\right. \\
& \mp \frac{1}{\xi \omega_{0}}\left[a^{4} \omega_{0}^{2}-2 a^{2} \xi\left(3+3 \omega_{0}+4 \omega_{1}\right) \omega_{0}\right. \\
& \left.\left.+4 \xi^{2}\left(21 \omega_{0}^{2}+\omega_{0}\left(2+26 \omega_{1}\right)+\left(1+3 \omega_{1}\right)^{2}\right)\right]^{1 / 2}\right]^{1 / 2}
\end{aligned}
$$

and for $v(53)$, we get

$v=-\frac{2 a^{2}\left\{4 \xi\left[\omega_{0}+\omega_{1}-\Omega\left(1+\omega_{0}\right)\right]+a^{2}\left[-\omega_{1}+\Omega\left(1+\omega_{0}\right)\right]\right\}}{\left(a^{2}-4 \xi\right)\left[a^{2}\left(1+\omega_{0}+2 \omega_{1}\right)-8 \xi\left(\omega_{0}+\omega_{1}\right)\right]}$.

From Eq. (56), we see that the Yukawa parameters $\mu_{1,2}$ are not related to the gravexciton/radion mass

$m_{\mathrm{rad}} \sim 1 / a$

and to the scalaron mass [23]

$$
\begin{aligned}
m_{\text {scal }} & =\frac{1}{\sqrt{5}}\left(-\frac{2 f^{\prime}\left(R_{0}\right)}{f^{\prime \prime}\left(R_{0}\right)}+R_{0}\right)^{1 / 2} \\
& =\frac{1}{\sqrt{5|\xi|}}\left(\frac{2|\xi|}{a^{2}}-\frac{|\xi|}{\xi}\right)^{1 / 2}
\end{aligned}
$$


by simple expressions. Nevertheless, if $|\xi| \sim a^{2}$, all masses are of the order of $1 / a: m_{\text {scal }} \sim m_{\text {rad }} \sim \mu_{1,2} \sim 1 / a$ (up to the natural assumption $\left.\omega_{0}, \omega_{1} \sim O(1)\right)$, so it appears interesting to consider a number of limiting cases to further explore such possible connections.

First, we study the case $|\xi| \gg a^{2}$. It can easily be seen that in this limit, the scalaron mass $m_{\text {scal }} \sim 1 / a$ (for any sign of $\xi$ ) is similar to the radion mass $m_{\text {rad }}$. Moreover, the parameters $\mu_{1,2}$ read

$$
\begin{aligned}
& \mu_{1,2} \approx \frac{1}{\sqrt{5}} \frac{1}{a}\left[-\frac{\left(1+\omega_{0}+3 \omega_{1}\right)}{\omega_{0}}\right. \\
&\left.\mp \frac{|\xi|}{\xi} \frac{1}{\omega_{0}}\left[21 \omega_{0}^{2}+\left(2+26 \omega_{1}\right) \omega_{0}+\left(1+3 \omega_{1}\right)^{2}\right]^{1 / 2}\right]^{1 / 2} .
\end{aligned}
$$

As we adopt the natural assumption $\omega_{0}, \omega_{1} \sim O(1)$, it is possible to see that both parameters $\mu_{1,2}$ as well as the scalaron and radion masses are defined by the radius $a$ of the sphere:

$\mu_{1,2} \sim m_{\mathrm{scal}} \sim m_{\mathrm{rad}} \sim \frac{1}{a}$.

Obviously, for $r \gg a$, the exponential terms in (46) and (47) can be dropped and for the parameter $v(57)$, we find

$v=\frac{a^{2}\left[\Omega\left(1+\omega_{0}\right)-\omega_{0}-\omega_{1}\right]}{4 \xi\left(\omega_{0}+\omega_{1}\right)}$.

Choosing $\omega_{0}=\bar{\omega}_{0}=-1$, the above expression implies

$-\frac{S_{D} \tilde{G}_{\mathcal{D}}}{V_{\text {int }}} \frac{a^{2}}{4 \xi}=4 \pi G_{N}, \quad \xi<0$,

which indeed corresponds to (55) since $R_{0}=-2 / a^{2}$ and again, $|\xi| \gg a^{2}$. Keeping in mind that $V_{\text {int }}=4 \pi a^{2}$, we realize that in this limit and for the particular case of $\omega_{0}=-1$, the relation between gravitational constants becomes independent of the internal space radius $a$ (up to some $O\left(a^{2}\right)$ correction term).

Now, we consider the reverse limiting case $|\xi| \ll a^{2}$. For the scalaron mass, we have $m_{\text {scal }} \sim(-1 / \xi)^{1 / 2}$, which is valid for negative values of $\xi$. For the parameters $\mu_{1,2}$ we obtain

$$
\begin{aligned}
\mu_{1,2} \approx & \frac{1}{\sqrt{10}} \frac{1}{a} \\
\times & {\left[-\frac{a^{2}}{\xi} \mp \frac{\left|\omega_{0}\right|}{\omega_{0}} \frac{a^{2}}{\xi}\left(1-\frac{\xi}{a^{2}} \frac{3+3 \omega_{0}+4 \omega_{1}}{\omega_{0}}\right)\right]^{1 / 2}, }
\end{aligned}
$$

which, for $\omega_{0}<0$, are reduced to the following:

$$
\begin{aligned}
& \mu_{1} \approx \frac{1}{\sqrt{10}} \frac{1}{a}\left(-\frac{3+3 \omega_{0}+4 \omega_{1}}{\omega_{0}}\right)^{1 / 2} \sim \frac{1}{a} \sim m_{\mathrm{rad}}, \\
& \mu_{2} \approx \frac{1}{\sqrt{5}} \frac{1}{a}\left(-\frac{a^{2}}{\xi}\right)^{1 / 2}=\frac{1}{\sqrt{5|\xi|}} \approx m_{\mathrm{scal}} .
\end{aligned}
$$

As for the important particular value $\omega_{0}=-1$, the inequality $3+3 \omega_{0}+4 \omega_{1}>0$ following from (65) leads to the condition $\omega_{1}>0$, similar to what has been obtained in $[31,32,34]$. On the other hand, Eq. (66) requires $\xi<0$. Since $a \gg \sqrt{|\xi|}$, $\mu_{1} \ll \mu_{2}$ and in Eqs. (46)-(49) we may drop the exponential functions with $\mu_{2}$. Consequently, given the solutions with a single Yukawa potential, we can apply the results of the inverse square law experiments [41] to get restrictions on the parameters of the model, similar to the ones we obtain at the very end of Sect. 4. For example, if $\beta_{1 A} / \kappa^{\prime}, \omega_{1} \sim O(1)$, then $a \lesssim 10^{-3} \mathrm{~cm} \mathrm{[41]} \mathrm{and} \mu_{1} \gtrsim 10^{-2} \mathrm{eV}$. This is, of course, a very rough estimate since the value of $\beta_{1 A}$ strongly depends on $\xi$ and $a$.

Let us now turn to the limit $a \rightarrow \infty$. The limiting values of the Yukawa parameters are found to be

$\mu_{1}=0, \quad \mu_{2}=\sqrt{\frac{-1}{5 \xi}}=m_{\mathrm{scal}}$,

and accordingly, the metric coefficients $A^{1}(r)$ and $B^{1}(r)$ have the forms

$$
\begin{aligned}
& A^{1}(r)=\sqrt{\frac{\pi}{2}} \frac{1}{r} \kappa^{\prime}\left[-\frac{2 \Omega+3}{2}+\frac{(2 \Omega-1)}{10} \exp \left(-\mu_{2} r\right)\right], \\
& B^{1}(r)=\sqrt{\frac{\pi}{2}} \frac{1}{r} \kappa^{\prime}\left[\frac{2 \Omega-1}{2}+\frac{(1-2 \Omega)}{10} \exp \left(-\mu_{2} r\right)\right] .
\end{aligned}
$$

As for the remaining corrections, we first renormalize $G^{1}(r)$ in the perturbed metric (11) and replace it by the term $a^{2} G^{1}(r)$. Then, the newly defined function in the limit $a \rightarrow \infty$ reads

$$
G^{1}(r)=\sqrt{\frac{\pi}{2}} \frac{1}{r} \kappa^{\prime}\left[-\frac{1+2 \Omega}{2}+\frac{1-2 \Omega}{10} \exp \left(-\mu_{2} r\right)\right] .
$$

Finally, the perturbed scalar curvature follows as

$$
R^{1}(r)=\sqrt{\frac{\pi}{2}} \frac{1}{r} \kappa^{\prime}\left[\frac{(1-2 \Omega)}{10 \xi} \exp \left(-\mu_{2} r\right)\right] .
$$

Expressions (68)-(71) exactly coincide with the formulas in paper [24] (for $D=5$ and up to the evident substitution $\Omega \rightarrow 2 \Omega$ ) devoted to nonlinear models with toroidal compactification of the internal space.

From (68) and (69), the condition $A^{1}(r \rightarrow \infty)=$ $B^{1}(r \rightarrow \infty)$ requires $\Omega=-1 / 2$, which is the EoS for black strings, and one consequently obtains

$v=1, \quad \frac{S_{D} \tilde{G}_{\mathcal{D}}}{V_{\text {int }}}=4 \pi G_{N}$. 
3.2 Equal roots: $\mu_{1}=\mu_{2} \equiv \mu>0$

In the case $\mu_{1}=\mu_{2} \equiv \mu$, Eqs. (43) and (44) can be rewritten as

$\tilde{A}^{1}(k)=\frac{\gamma_{1 A}}{k^{2}+\mu^{2}}+\frac{\gamma_{2 A}}{\left(k^{2}+\mu^{2}\right)^{2}}+\frac{\gamma_{3}}{k^{2}}$,
$\tilde{B}^{1}(k)=\frac{\gamma_{1 B}}{k^{2}+\mu^{2}}+\frac{\gamma_{2 B}}{\left(k^{2}+\mu^{2}\right)^{2}}+\frac{\gamma_{3}}{k^{2}}$,

and

$\tilde{G}^{1}(k)=\frac{\gamma_{1 G}}{k^{2}+\mu^{2}}+\frac{\gamma_{2 G}}{\left(k^{2}+\mu^{2}\right)^{2}}$,

$\tilde{R}^{1}(k)=\frac{\gamma_{1 R}}{k^{2}+\mu^{2}}+\frac{\gamma_{2 R}}{\left(k^{2}+\mu^{2}\right)^{2}}$,

where we have introduced the coefficients

$$
\begin{aligned}
\gamma_{1 A(B)} & \equiv \frac{\alpha_{1 A(B)} \mu^{4}-\alpha_{3}}{\alpha_{4} \mu^{4}}, \\
\gamma_{2 A(B)} & \equiv \frac{\alpha_{2 A(B)} \mu^{2}-\alpha_{1 A(B)} \mu^{4}-\alpha_{3}}{\alpha_{4} \mu^{2}}, \\
\gamma_{3} & \equiv \frac{\alpha_{3}}{\alpha_{4} \mu^{4}}, \\
\gamma_{1 G(R)} & \equiv \frac{\alpha_{1 G(R)}}{\alpha_{4}}, \\
\gamma_{2 G(R)} & \equiv \frac{\alpha_{2 G(R)}-\alpha_{1 G(R)} \mu^{2}}{\alpha_{4}} .
\end{aligned}
$$

The explicit expression for the root now reads

$$
\mu=\sqrt{\frac{\alpha_{5}}{2 \alpha_{4}}}=\sqrt{-\frac{1}{5}\left(\frac{f^{\prime}\left(R_{0}\right)}{f^{\prime \prime}\left(R_{0}\right)}+\frac{\left(1+3 \omega_{0}+3 \omega_{1}\right)}{a^{2} \omega_{0}}\right)} .
$$

In contrast with Eqs. (43) and (44), where the Newtonian and Yukawa potentials are the only contributions to the metric coefficients, the term $1 /\left(k^{2}+\mu^{2}\right)^{2}$ in (73) and (74) provides new contribution in the form of a pure exponential potential. Therefore, in position space we get

$$
\begin{aligned}
& A^{1}(r)=\sqrt{\frac{\pi}{2}} \frac{1}{r}\left[\gamma_{3}+\left(\gamma_{1 A}+\frac{r}{2 \mu} \gamma_{2 A}\right) \exp (-\mu r)\right], \\
& B^{1}(r)=\sqrt{\frac{\pi}{2}} \frac{1}{r}\left[\gamma_{3}+\left(\gamma_{1 B}+\frac{r}{2 \mu} \gamma_{2 B}\right) \exp (-\mu r)\right], \\
& G^{1}(r)=\sqrt{\frac{\pi}{2}} \frac{1}{r}\left(\gamma_{1 G}+\frac{r}{2 \mu} \gamma_{2 G}\right) \exp (-\mu r), \\
& R^{1}(r)=\sqrt{\frac{\pi}{2}} \frac{1}{r}\left(\gamma_{1 R}+\frac{r}{2 \mu} \gamma_{2 R}\right) \exp (-\mu r) .
\end{aligned}
$$

Taking into account (76), we can easily see that $\gamma_{3}=$ $\alpha_{3} /\left(\alpha_{4} \mu^{4}\right)=\alpha_{3} / \alpha_{6}=\beta_{3}$. Thus, for the limiting case $r \rightarrow \infty$, we drop the exponential terms in the above set and reproduce Eqs. (52)-(55) to conclude that the corresponding physical interpretations therein hold valid also for the model with equal roots. For finite $r$, the Yukawa potential receives a "corrupted" prefactor presented as a combination of the $1 / r$ term and a second term independent of the three-dimensional distance $r$.

Example: $f(R)=R+\xi R^{2}$

Substituting $f(R)=R+\xi R^{2}$ in (76), we obtain

$\mu=\frac{1}{\sqrt{|\xi|}}\left(-\frac{|\xi|}{a^{2}} \frac{1+\omega_{0}+3 \omega_{1}}{5 \omega_{0}}-\frac{1}{10} \frac{|\xi|}{\xi}\right)^{1 / 2}$.

Following the reasoning in the previous subsection, we again consider two limiting cases $|\xi| \gg a^{2}$ and $|\xi| \ll a^{2}$, respectively. As we have already seen, for $|\xi| \gg a^{2}$, the scalaron mass becomes inversely proportional to the internal space radius: $m_{\text {scal }} \sim 1 / a$. In this limit, the parameter $\mu$ reads

$\mu \approx \frac{1}{a}\left(\frac{1+\omega_{0}+3 \omega_{1}}{-5 \omega_{0}}\right)^{1 / 2} \sim m_{\mathrm{scal}} \sim m_{\mathrm{rad}} \sim \frac{1}{a}$,

and all characteristic masses appear to be defined by the radius of the sphere. Clearly, the argument of the square root must be positive. For example, in the important case $\omega_{0}=\bar{\omega}_{0}=-1$, the EoS parameter $\omega_{1}$ should be positive analogous to the conclusion following from equation (65).

In the limit $|\xi| \ll a^{2}$ the scalaron mass $m_{\text {scal }} \sim 1 / \sqrt{|\xi|}, \xi<$ 0 . For the parameter $\mu$, we obtain

$\mu \approx \sqrt{\frac{1}{10|\xi|}} \sim m_{\mathrm{scal}}$,

and deduce that it is proportional to the scalaron mass.

\section{The case $f^{\prime \prime}\left(R_{0}\right)=0, \omega_{0} \neq 0$}

In this section, we consider the case of a zero value of the second derivative $f^{\prime \prime}\left(R_{0}\right)=0$. Nevertheless, we demand that the first derivative is still non-vanishing: $f^{\prime}\left(R_{0}\right) \neq 0$. A particular example of such case is the linear model where $f(R)=R+2 \kappa \Lambda_{6}$. In what follows, the EoS parameters $\omega_{0}$ and $\omega_{1}$ are not equal to the background values $\bar{\omega}_{0}$ and $\bar{\omega}_{1}$ in general and we assume the additional inequality $\omega_{0} \neq$ 0. Then, as it follows from Eqs. (37), (38), (40), and (41), coefficients $\alpha_{1 A}=\alpha_{1 B}=\alpha_{4}=\alpha_{1 G}=0$. For the metric coefficients (36) and (39), we get

$$
\begin{gathered}
\tilde{A}^{1}(k)=\frac{\left(\alpha_{2 A} / \alpha_{5}\right) k^{2}+\left(\alpha_{3} / \alpha_{5}\right)}{k^{2}\left[k^{2}+\left(\alpha_{6} / \alpha_{5}\right)\right]}, \\
\tilde{B}^{1}(k)=\frac{\left(\alpha_{2 B} / \alpha_{5}\right) k^{2}+\left(\alpha_{3} / \alpha_{5}\right)}{k^{2}\left[k^{2}+\left(\alpha_{6} / \alpha_{5}\right)\right]},
\end{gathered}
$$

and

$$
\begin{aligned}
\tilde{G}^{1}(k) & =\frac{\left(\alpha_{2 G} / \alpha_{5}\right)}{k^{2}+\left(\alpha_{6} / \alpha_{5}\right)}, \\
\tilde{R}^{1}(k) & =\frac{\left(\alpha_{1 R} / \alpha_{5}\right) k^{2}+\left(\alpha_{2 R} / \alpha_{5}\right)}{k^{2}+\left(\alpha_{6} / \alpha_{5}\right)} .
\end{aligned}
$$


It is also possible to re-express the above solutions in the form of (43) and (44). For example,

$$
\begin{aligned}
& \tilde{A}^{1}(k)=\frac{\left(\alpha_{2 A} / \alpha_{5}\right)-\left(\alpha_{3} / \alpha_{5}\right) \mu^{-2}}{k^{2}+\mu^{2}}+\frac{\left(\alpha_{3} / \alpha_{5}\right) \mu^{-2}}{k^{2}}, \\
& \tilde{R}^{1}(k)=\frac{\alpha_{1 R}}{\alpha_{5}}+\frac{\left(\alpha_{2 R} / \alpha_{5}\right)-\mu^{2}\left(\alpha_{1 R} / \alpha_{5}\right)}{k^{2}+\mu^{2}},
\end{aligned}
$$

where $\mu^{2} \equiv \alpha_{6} / \alpha_{5}$. Choosing $\mu>0$, the solutions in the position space read

$$
\begin{aligned}
A^{1}(r)= & \sqrt{\frac{\pi}{2}} \frac{1}{r}\left[\frac{\alpha_{3}}{\alpha_{6}}+\left(\frac{\alpha_{2 A}}{\alpha_{5}}-\frac{\alpha_{3}}{\alpha_{6}}\right) \exp (-\mu r)\right], \\
B^{1}(r)= & \sqrt{\frac{\pi}{2}} \frac{1}{r}\left[\frac{\alpha_{3}}{\alpha_{6}}+\left(\frac{\alpha_{2 B}}{\alpha_{5}}-\frac{\alpha_{3}}{\alpha_{6}}\right) \exp (-\mu r)\right], \\
G^{1}(r)= & \sqrt{\frac{\pi}{2}} \frac{1}{r}\left[\left(\frac{\alpha_{2 G}}{\alpha_{5}}\right) \exp (-\mu r)\right], \\
R^{1}(r)= & (2 \pi)^{3 / 2} \delta(\mathbf{r}) \frac{\alpha_{1 R}}{\alpha_{5}} \\
& +\sqrt{\frac{\pi}{2}} \frac{1}{r}\left[\left(\frac{\alpha_{2 R}}{\alpha_{5}}-\frac{\alpha_{1 R}}{\alpha_{5}} \cdot \frac{\alpha_{6}}{\alpha_{5}}\right) \exp (-\mu r)\right] .
\end{aligned}
$$

The first term on the right-hand side (RHS) of (91) follows from the constant $\alpha_{1 R} / \alpha_{5}$ in (87).

To analyze these expressions, we need to define the first derivative $f^{\prime}\left(R_{0}\right)$. For this purpose, we consider the significant case of $f^{\prime}\left(R_{0}\right)=1$, which yields

$$
\begin{aligned}
A^{1}(r)= & \sqrt{\frac{\pi}{2}} \frac{1}{r} \kappa^{\prime}\left\{\frac{2\left[\Omega\left(1+\omega_{0}\right)-\omega_{1}\right]}{\left(1+\omega_{0}+2 \omega_{1}\right)}\right. \\
& -\left[\frac{3+2 \Omega}{2}+\frac{2\left[\Omega\left(1+\omega_{0}\right)-\omega_{1}\right]}{\left(1+\omega_{0}+2 \omega_{1}\right)}\right] \\
& \left.\times \exp \left(-\sqrt{-\frac{1}{2} \frac{\left(1+\omega_{0}+2 \omega_{1}\right)}{\omega_{0}}} \frac{r}{a}\right)\right\}, \\
B^{1}(r)= & \sqrt{\frac{\pi}{2}} \frac{1}{r} \kappa^{\prime}\left\{\frac{2\left[\Omega\left(1+\omega_{0}\right)-\omega_{1}\right]}{\left(1+\omega_{0}+2 \omega_{1}\right)}\right. \\
& -\left[\frac{1-2 \Omega}{2}+\frac{2\left[\Omega\left(1+\omega_{0}\right)-\omega_{1}\right]}{\left(1+\omega_{0}+2 \omega_{1}\right)}\right] \\
& \left.\times \exp \left(-\sqrt{-\frac{1}{2} \frac{\left(1+\omega_{0}+2 \omega_{1}\right)}{\omega_{0}}} \frac{r}{a}\right)\right\}, \\
G^{1}(r)= & -\sqrt{\frac{\pi}{2}} \frac{1}{r} \kappa^{\prime} a^{2}\left(\frac{1}{2}+\Omega\right) \\
& \times \exp \left(-\sqrt{-\frac{1}{2} \frac{\left(1+\omega_{0}+2 \omega_{1}\right)}{\omega_{0}}} \frac{r}{a}\right), \\
R^{1}(r)= & -\kappa^{\prime}(2 \pi)^{3 / 2} \delta(\mathbf{r})\left(\frac{1}{2}-\Omega\right) \\
& +\sqrt{\frac{\pi}{2}} \frac{1}{r} \kappa^{\prime}\left[\frac{\left(2 \omega_{1}+3 \omega_{0}-1\right)(1+2 \Omega)}{4 a^{2} \omega_{0}}\right.
\end{aligned}
$$

$$
\frac{\left(1+\omega_{0}+2 \omega_{1}\right)}{\omega_{0}}<0,
$$

which may be satisfied either for $\omega_{0}<0$ with $1+\omega_{0}$ $+2 \omega_{1}>0$, or for $\omega_{0}>0$ with $1+\omega_{0}+2 \omega_{1}<0$. The former combination agrees with the above discussed case where we have set $\omega_{0}=-1$ and additionally, introduces the 
constraint $\omega_{1}>0$ which is the necessary condition for the internal space stabilization [31,32,34].

Equation (92) shows that in the case of arbitrary $\Omega \neq$ $-1 / 2$, the gravitational potential acquires a Yukawa correction term. As the inverse square law experiments [41] put restrictions on the parameters of such corrections, following the reasoning in [32], we may actually obtain an upper limit on the size of the extra dimensions here. For example, if $\omega_{0}=-1$ and for the natural assumption $|\Omega|, \omega_{1} \sim O(1)$, the maximal value of the internal space radius $a_{\max } \sim 10^{-3} \mathrm{~cm}$ [41]. Given that the radius of the Sun $r_{\odot} \sim 7 \times 10^{10} \mathrm{~cm}$, we can drop the Yukawa correction terms in such models while studying the gravitational effects (the deflection of light and time delay of radar echoes) with very high accuracy.

\section{The case $\omega_{0}=0$}

We are now interested in the case $\omega_{0}=0$. Since $\omega_{0} \neq \bar{\omega}_{0}=$ -1 , the background perfect fluid is nonlinear. According to Eq. (18), a zero value of $\omega_{0}$ results in $\delta p_{0}=0$. The steps through (19)-(28) for this special case show that

$0=-f^{\prime}\left(R_{0}\right) \frac{G^{1}}{a^{4}}$,

which, for $f^{\prime}\left(R_{0}\right) \neq 0$, implies

$G^{1}=0$.

Therefore, Eqs. (25), (26), (19), (21), and (28) read

$$
\begin{aligned}
& \frac{1}{2} f^{\prime}\left(R_{0}\right)\left(-A^{1}+B^{1}\right)-f^{\prime \prime}\left(R_{0}\right) R_{1}=0, \\
& \frac{f^{\prime}\left(R_{0}\right)}{2}\left(\Delta_{3} B^{1}+R_{1}\right)+\left(\Delta_{3} R_{1}\right) f^{\prime \prime}\left(R_{0}\right)=0, \\
& \frac{f^{\prime}\left(R_{0}\right)}{2}\left(\Delta_{3} A^{1}-R_{1}\right)-\left(\Delta_{3} R_{1}\right) f^{\prime \prime}\left(R_{0}\right)=\kappa\left(\delta \varepsilon+\hat{\rho} c^{2}\right), \\
& f^{\prime}\left(R_{0}\right) R_{1}+f^{\prime \prime}\left(R_{0}\right) \frac{2 R_{1}}{a^{2}}+2\left(\Delta_{3} R_{1}\right) f^{\prime \prime}\left(R_{0}\right) \\
& =2 \kappa\left(\delta p_{1}+\Omega \hat{\rho} c^{2}\right) \\
& -2 f^{\prime}\left(R_{0}\right) R_{1}+f^{\prime \prime}\left(R_{0}\right)\left(-\frac{2}{a^{2}} R_{1}-5 \Delta_{3} R_{1}\right) \\
& =\kappa\left[\delta \varepsilon-2 \delta p_{1}+\hat{\rho} c^{2}(1-2 \Omega)\right]
\end{aligned}
$$

respectively.

$$
5.1 f^{\prime \prime}\left(R_{0}\right) \neq 0
$$

We now demand that $f^{\prime \prime}\left(R_{0}\right) \neq 0$ and hence, exclude the linear model $f(R)=R+2 \kappa \Lambda_{6}$. Combining Eqs. (106) and (107), it is possible to obtain an expression for $R_{1}$, that is

$$
R_{1}=2 \kappa a^{2}\left[\frac{\delta \varepsilon\left(1+3 \omega_{1}\right)+\hat{\rho} c^{2}(1+3 \Omega)}{6 f^{\prime \prime}\left(R_{0}\right)+a^{2} f^{\prime}\left(R_{0}\right)}\right]
$$

Similarly, for $\Delta_{3} R_{1}$, the same set yields

$$
\begin{aligned}
\frac{\Delta_{3} R_{1}}{\kappa}= & -\frac{2 f^{\prime \prime}\left(R_{0}\right)\left(\delta \varepsilon+\hat{\rho} c^{2}\right)}{f^{\prime \prime}\left(R_{0}\right)\left[6 f^{\prime \prime}\left(R_{0}\right)+a^{2} f^{\prime}\left(R_{0}\right)\right]} \\
& -\frac{a^{2} f^{\prime}\left(R_{0}\right)\left[\delta \varepsilon\left(1+2 \omega_{1}\right)+\hat{\rho} c^{2}(1+2 \Omega)\right]}{f^{\prime \prime}\left(R_{0}\right)\left[6 f^{\prime \prime}\left(R_{0}\right)+a^{2} f^{\prime}\left(R_{0}\right)\right]} .
\end{aligned}
$$

For further calculations, we assume a relation between $\delta \varepsilon$ and $\hat{\rho} c^{2}$ in the form

$\delta \varepsilon=\zeta \hat{\rho} c^{2}$,

which is the fine tuning condition. The parameter $\zeta$ here is to be specified in the later steps. Substituting (108) and (109) into Eqs. (104) and (105), we obtain

$$
\begin{aligned}
& \Delta_{3} A^{1}(r)=2 \kappa \hat{\rho} c^{2} \\
& \times\left[\frac{4 f^{\prime \prime}\left(R_{0}\right)(1+\zeta)+a^{2} f^{\prime}\left(R_{0}\right)\left[1+\Omega+\zeta\left(1+\omega_{1}\right)\right]}{f^{\prime}\left(R_{0}\right)\left[6 f^{\prime \prime}\left(R_{0}\right)+a^{2} f^{\prime}\left(R_{0}\right)\right]}\right],
\end{aligned}
$$

and

$$
\begin{aligned}
\Delta_{3} B^{1}(r)= & 2 \kappa \hat{\rho} c^{2} \\
& \times\left[\frac{2 f^{\prime \prime}\left(R_{0}\right)(1+\zeta)-a^{2} f^{\prime}\left(R_{0}\right)\left(\Omega+\zeta \omega_{1}\right)}{f^{\prime}\left(R_{0}\right)\left[6 f^{\prime \prime}\left(R_{0}\right)+a^{2} f^{\prime}\left(R_{0}\right)\right]}\right] .
\end{aligned}
$$

The overall factor $2 \kappa \hat{\rho} c^{2}$ on the RHS of the above equations entails a delta-shaped function, therefore, one may immediately deduce that solutions $A^{1}(r)$ and $B^{1}(r)$ will both be proportional to $1 / r$. Since we will eventually demand that $A^{1}(r \rightarrow \infty)=B^{1}(r \rightarrow \infty)$, it turns out useful to set (111) and (112) equal to each other from the very beginning. This requires

$\zeta=-\frac{2 f^{\prime \prime}\left(R_{0}\right)+a^{2} f^{\prime}\left(R_{0}\right)(1+2 \Omega)}{2 f^{\prime \prime}\left(R_{0}\right)+a^{2} f^{\prime}\left(R_{0}\right)\left(1+2 \omega_{1}\right)}$,

hence, $R_{1}$ in (108) reads

$R_{1}=2 \kappa a^{2}\left[\frac{\hat{\rho} c^{2}\left(\Omega-\omega_{1}\right)}{2 f^{\prime \prime}\left(R_{0}\right)+a^{2} f^{\prime}\left(R_{0}\right)\left(1+2 \omega_{1}\right)}\right]$.

In order to get the PPN parameter $\gamma=B^{1} / A^{1}=1$ as in General Relativity (which is in very good agreement with the gravitational tests in the Solar system), from Eq. (103), we need to impose $R_{1}=0$. For vanishing $R_{1}$, Eqs. (113) and (114) imply

$\Omega=\omega_{1}, \quad \zeta=-1$. 
In return, (111) and (112) take on the form

$\Delta_{3} A^{1}(r)=\Delta_{3} B^{1}(r)=0$,

with the trivial solution

$A^{1}=B^{1}=0$,

taking into account the boundary condition $A^{1}(r \rightarrow \infty)=$ $B^{1}(r \rightarrow \infty)=0$. The physical reason for this trivial solution is that the matter sources $\delta \varepsilon$ and $\hat{\rho} c^{2}$ on the RHS of Eqs. (105)-(107) mutually cancel: $\delta \varepsilon+\hat{\rho} c^{2}=0$.

\section{$5.2 f^{\prime \prime}\left(R_{0}\right)=0$}

The linear model $f(R)=R+2 \kappa \Lambda_{6}$ is a particular example of the case where $f^{\prime \prime}\left(R_{0}\right)=0$. Combined with the fine tuning condition (110), from (106) and (113), we get

$R_{1}=2 \kappa \hat{\rho} c^{2}\left(\frac{\Omega+\zeta \omega_{1}}{f^{\prime}\left(R_{0}\right)}\right)$,

and

$\zeta=-\frac{1+2 \Omega}{1+2 \omega_{1}}$.

For this value of $\zeta$, Eq. (107) is reduced to Eq. (106). In the case $\Omega=0$, we obtain the relation $\zeta=-1 /\left(1+2 \omega_{1}\right)$, which exactly reproduces the corresponding results in paper [38]. On the other hand, for black branes, i.e., for $\Omega=-1 / 2$, we find $\zeta=0$ that leads to $\delta \varepsilon=0$. For arbitrary $\Omega$, Eq. (103) demonstrates that the relation $A^{1}=B^{1}$ is satisfied automatically. As it follows from Eqs. (104) and (105), both of these corrections satisfy the equations

$\Delta_{3} A^{1}(r)=\Delta_{3} B^{1}(r)=\kappa \hat{\rho} c^{2} v$,

with solutions

$A^{1}(r)=B^{1}(r)=-\frac{2}{c^{2}} \frac{1}{4 \pi} \frac{S_{D} \tilde{G}_{\mathcal{D}}}{V_{\mathrm{int}}} \frac{m}{r} v$,

where

$v \equiv \frac{2\left(\omega_{1}-\Omega\right)}{f^{\prime}\left(R_{0}\right)\left(1+2 \omega_{1}\right)}$.

The condition $A^{1}=B^{1}=2 \varphi_{N} / c^{2}=-2 G_{N} m /\left(c^{2} r\right)$ now imposes

$\frac{S_{D} \tilde{G}_{\mathcal{D}}}{V_{\text {int }}} v=4 \pi G_{N}$.

For $f^{\prime}\left(R_{0}\right)=1$, we see that black branes $\operatorname{EoS} \Omega=-1 / 2$ restores the conventional relation $S_{D} \tilde{G}_{\mathcal{D}} / V_{\text {int }}=4 \pi G_{N}$. Obviously, the relation $A^{1}(r)=B^{1}(r)$ in (121) guarantees that the PPN parameter $\gamma=B^{1}(r) / A^{1}(r)=1$ and exactly coincides with the value for $\gamma$ in General Relativity.

\section{Conclusion}

In the present paper we have studied multidimensional nonlinear $f(R)$ models with spherical compactification of the internal space. The background space-time is endowed with perfect fluid which makes the internal space compact and curved in the form of a two-dimensional sphere of radius $a$. Similar to the linear case [31-35], this fluid has the vacuumlike EoS parameter $\bar{\omega}_{0}=-1$ in the external space. In the internal space, the EoS parameter $\bar{\omega}_{1}$ depends on the form of $f(R)$. Then, we have perturbed this background by a massive gravitating source which is pressureless in the external space but has an arbitrary EoS parameter $\Omega$ in the internal space. We have considered a nonlinear perfect fluid, i.e., we have assumed that the parameters $\omega_{0}=\delta p_{0} / \delta \varepsilon \neq \bar{\omega}_{0}$ and $\omega_{1}=\delta p_{1} / \delta \varepsilon \neq \bar{\omega}_{1}$, where $\delta p_{0}\left(\delta p_{1}\right)$ denotes the fluctuation of the perfect fluid pressure in the external (internal) space and $\delta \varepsilon$ is the fluctuation in the corresponding energy density.

Then we have obtained the linearized Einstein equations for the perturbed metric coefficients. We have shown that all Einstein equations are reduced to the system of four master equations (25) and (31)-(33). Despite the rather complex form of these equations, we have found the exact solutions in the general case $f^{\prime \prime}\left(R_{0}\right) \neq 0$, where $R_{0}$ is the scalar curvature of the background space-time and prime denotes differentiation with respect to the scalar curvature $R$. It was then revealed that the solutions are valid only for the case $\omega_{0} \neq 0$. As is well known, a characteristic feature of nonlinear $f(R)$ models is the presence of the scalar degree of freedom, dubbed the scalaron. On the other hand, multidimensional models also have a scalar degree of freedom - the gravexciton/radion - due to the fluctuations of the internal space volume. Here, we have shown that the perturbed metric coefficients acquire correction terms in the form of the sum of two Yukawa potentials with Yukawa masses $\mu_{1}$ and $\mu_{2}$ (51). We have demonstrated that masses $\mu_{1,2}$ have a rather complex form which cannot be related to the mass $m_{\text {scal }}$ of scalaron and $m_{\text {rad }}$ of gravexciton/radion by simple expressions. Nevertheless, for the concrete model $f(R)=R+\xi R^{2}$, we were able to trace the relationship in a number of limiting cases. For example, when $|\xi| \sim a^{2}$, all masses appear to be of the same order of magnitude: $\mu_{1}, \mu_{2}, m_{\mathrm{scal}}, m_{\mathrm{rad}} \sim 1 / a$. A similar situation takes place for $|\xi| \gg a^{2}$. For $|\xi| \ll a^{2}$, we get $\mu_{1} \sim m_{\text {rad }}$ and $\mu_{2} \sim m_{\text {scal }}$. Another limiting case $a \rightarrow \infty$ corresponds to $\mu_{1}=0, \mu_{2}=m_{\text {scal }}$ and all the derived expressions for the metric coefficients exactly coincide with the formulas in paper [24], devoted to nonlinear models with toroidal compactification of the internal space. It has been demonstrated that the black string $\operatorname{EoS} \Omega=-1 / 2$ ensures the fulfilment of the condition $A^{1}(r \rightarrow \infty)=B^{1}(r \rightarrow \infty)$.

In the degenerated case with a single Yukawa mass, $\mu_{1}=\mu_{2}=\mu$, metric coefficients no longer have the previ- 
ously obtained form of combined Yukawa potentials. Instead, we have shown that the solutions have the form of a single Yukawa potential with a "corrupted" prefactor (in front of the exponential function) which, in addition to the standard $1 / r$ term, contains a contribution independent of the threedimensional distance $r$.

We have also investigated the class of models with $f^{\prime \prime}\left(R_{0}\right)=0$ (to which the specific linear model $f(R)=$ $R+2 \kappa \Lambda_{6}$ belongs) with nonlinear background perfect fluid for the condition $\omega_{0} \neq 0$. The linear gravity models with spherical compactification involving linear background perfect fluid were investigated previously in [31-33]. In this work, we have generalized these models to the case of an arbitrary nonlinear background perfect fluid. In agreement with the conclusions of previous papers, it has been shown that there are two possible situations in which the gravitational tests in the Solar system (the deflection of light, time delay of radar echoes and perihelion shift) are satisfied and the corresponding constraints on the PPN parameter $\gamma$ are met. First, it takes place for a sufficiently large value of the gravexciton/radion mass $m_{\text {rad }} \sim 1 / a$, for which one may drop the Yukawa correction term in the metric coefficients. In this case, the EoS parameter $\Omega$ (that defines the pressure of the gravitating source in the internal space) can be arbitrary. Second, for the case $\Omega=-1 / 2[20,21,30]$, the Yukawa correction terms vanish automatically and arbitrary values of the mass $m_{\text {rad }}$ are allowed.

Finally, we have considered the case of zero speed of sound for the nonlinear perfect fluid in the external space: $\omega_{0}=0$. In the nonlinear gravity model with $f^{\prime \prime}\left(R_{0}\right) \neq 0$, the condition $\gamma=1$ for the PPN parameter $\gamma$ led to the trivial solution (117) due to mutual cancellation of the matter sources on the RHS of the linearized Einstein equations (105)-(107): $\delta \varepsilon+\hat{\rho} c^{2}=0$. In the case $f^{\prime \prime}\left(R_{0}\right)=0$, we have shown that the condition $\gamma=1$ is automatically satisfied for arbitrary values of the $\operatorname{EoS}$ parameter $\Omega$. Yet, it is important to stress that only for the black branes/strings with $\Omega=-1 / 2$ one may ensure the absence of fluctuations in the background perfect fluid energy density, i.e. $\delta \varepsilon=0$, which indeed translates into the absence of nonphysical coat around the gravitating mass [42].

The validity of our solutions is limited at two opposite scales. First, we consider the weak field limit in which the gravitational field is weak and peculiar velocities of test masses are much less than the speed of light. Consequently, the results cease to hold in the vicinity of such relativistic astrophysical objects as black holes and neutron stars. Second, our approach is violated on scales where the cosmological expansion must be taken into account. The range of validity, in this case, depends on the masses of the astrophysical objects. For example, for a typical galaxy of a mass of the Milky Way, it corresponds to an order of $1 \mathrm{Mpc}[43,44]$. In the four-dimensional case, an approach that allows to consider gravitational interaction on all scales (from astrophysical to cosmological ones) was proposed in paper [45].

Data Availability Statement This manuscript has no associated data or the data will not be deposited. [Authors' comment: Data sharing not applicable to this article as no datasets were generated or analysed during the current study.]

Open Access This article is licensed under a Creative Commons Attribution 4.0 International License, which permits use, sharing, adaptation, distribution and reproduction in any medium or format, as long as you give appropriate credit to the original author(s) and the source, provide a link to the Creative Commons licence, and indicate if changes were made. The images or other third party material in this article are included in the article's Creative Commons licence, unless indicated otherwise in a credit line to the material. If material is not included in the article's Creative Commons licence and your intended use is not permitted by statutory regulation or exceeds the permitted use, you will need to obtain permission directly from the copyright holder. To view a copy of this licence, visit http://creativecomm ons.org/licenses/by/4.0/.

Funded by $\mathrm{SCOAP}^{3}$.

\section{Appendix: Perturbed Ricci tensor}

In this appendix we present the nonzero components of the Ricci tensor corresponding to the metric (11). Obviously, the off-diagonal components $R_{0 i}(i=1,2,3,4,5)$ vanish for static metrics. Using the results of the paper [31], we can easily find that the $R_{5 i}$ components for $i=1,2,3,4$ and $R_{4 i}$ components for $i=1,2,3$ are also equal to zero. For the diagonal components we have

$$
\begin{aligned}
R_{00} & =\frac{1}{2} \Delta_{3} A^{1}=R_{00}^{(1)} \\
R_{i i} & =\frac{1}{2} \Delta_{3} B^{1}+\frac{1}{2}\left[-A^{1}+B^{1}+2 \frac{G^{1}}{a^{2}}\right]_{x^{i} x^{i}}=R_{i i}^{(1)} \\
i & =1,2,3 \\
R_{44} & =1+\frac{1}{2} \Delta_{3} G^{1}=1+R_{44}^{(1)} \\
R_{55} & =\left(1+\frac{1}{2} \Delta_{3} G^{1}\right) \sin ^{2} \xi=\sin ^{2} \xi+R_{55}^{(1)}
\end{aligned}
$$

where $\Delta_{3}=\partial^{2} / \partial x^{2}+\partial^{2} / \partial y^{2}+\partial^{2} / \partial z^{2}$ is the threedimensional Laplace operator. The remaining off-diagonal components are

$$
\begin{aligned}
& R_{12}=\frac{1}{2}\left[-A^{1}+B^{1}+2 \frac{G^{1}}{a^{2}}\right]_{x y}=R_{12}^{(1)} \\
& R_{13}=\frac{1}{2}\left[-A^{1}+B^{1}+2 \frac{G^{1}}{a^{2}}\right]_{x z}=R_{13}^{(1)} \\
& R_{23}=\frac{1}{2}\left[-A^{1}+B^{1}+2 \frac{G^{1}}{a^{2}}\right]_{y z}=R_{23}^{(1)} .
\end{aligned}
$$


The scalar curvature perturbation is a function of the external coordinates only: $R_{1}=R_{1}(x, y, z)$. The only non-zero derivatives are

$\left(R_{1}\right)_{; i ; k} \approx \frac{\partial^{2} R_{1}}{\partial x^{i} \partial x^{k}}$ for $i, k=1,2,3$.

Then,

$\left(R_{1}\right)_{; m ; n} g^{(0) m n}=-\Delta_{3} R_{1}$.

\section{References}

1. T.P. Sotiriou, V. Faraoni, $f(R)$ theories of gravity. Rev. Mod. Phys. 82, 451-497 (2010). arXiv:0805.1726 [gr-qc]

2. A. De Felice, S. Tsujikawa, $f(R)$ theories. LRR 13, 3 (2010). arXiv: 1002.4928 [gr-qc]

3. S. Capozziello, M. De Laurentis, Extended theories of gravity. Phys. Rep. 509, 167-321 (2011). arXiv:1108.6266 [gr-qc]

4. S. Nojiri, S.D. Odintsov, Unified cosmic history in modified gravity: from $F(R)$ theory to Lorentz non-invariant models. Phys. Rep. 505, 59-144 (2011). arXiv:1011.0544 [gr-qc]

5. T. Clifton, P.G. Ferreira, A. Padilla, C. Skordis, Modified gravity and cosmology. Phys. Rep. 513, 1-189 (2012). arXiv:1106.2476 [astro-ph.CO]

6. S. Nojiri, S.D. Odintsov, V.K. Oikonomou, Modified gravity theories on a nutshell: Inflation, bounce and late-time evolution. Phys. Rep. 692, 1-104 (2017). arXiv:1705.11098 [gr-qc]

7. D. Bailin, A. Love, Kaluza-Klein theories. Rep. Prog. Phys. 50, 1087-1170 (1987)

8. J.M. Overduin, P.S. Wesson, Kaluza-Klein gravity. Phys. Rep. 283, 303-380 (1997). arXiv:gr-qc/9805018

9. J. Polchinski, String Theory, vol. 2 (Cambridge University Press, Cambridge, 1998)

10. P.S. Wesson, Space-Time-Matter (World Scientific, Singapore, 1999)

11. R. Maartens, K. Koyama, Brane-world gravity. Living Rev. Relat. 13, 5 (2010). arXiv:1004.3962 [hep-th]

12. U. Günther, P. Moniz, A. Zhuk, Asymptotical AdS space from nonlinear gravitational models with stabilized extra dimensions. Phys. Rev. D 66, 044014 (2002). arXiv:hep-th/0205148

13. U. Günther, P. Moniz, A. Zhuk, Nonlinear multidimensional cosmological models with form fields: Stabilization of extra dimensions and the cosmological constant problem. Phys. Rev. D 68, 044010 (2003). arXiv:hep-th/0303023

14. T. Saidov, A. Zhuk, AdS non-linear curvature-squared and curvature-quartic multidimensional $(D=8)$ gravitational models with stabilized extra dimensions. Grav. Cosmol. 12, 253-261 (2006). arXiv:hep-th/0604131

15. T. Saidov, A. Zhuk, $1 / R$ multidimensional gravity with form-fields: stabilization of extra dimensions, cosmic acceleration and domain walls. Phys. Rev. D 75, 084037 (2007). arXiv:hep-th/0612217

16. T. Saidov, A. Zhuk, On the problem of inflation in nonlinear multidimensional cosmological models. Phys. Rev. D 79, 024025 (2009). arXiv:0809.5226 [hep-th]

17. T. Saidov, A. Zhuk, Bouncing inflation in nonlinear $R^{2}+R^{4}$ gravitational model. Phys. Rev. D 81, 124002 (2010). arXiv:1002.4138 [hep-th]

18. H. Nakada, S.V. Ketov, Inflation from higher dimensions. Phys. Rev. D 96, 123530 (2017). arXiv:1710.02259 [hep-th]

19. M. Eingorn, A. Zhuk, Classical tests of multidimensional gravity: negative result. Class. Quantum Grav. 27, 205014 (2010). arXiv:1003.5690 [gr-qc]
20. M. Eingorn, A. Zhuk, Kaluza-Klein models: can we construct a viable example? Phys. Rev. D 83, 044005 (2011). arXiv: 1010.5740 [gr-qc]

21. M. Eingorn, O. de Medeiros, L. Crispino, A. Zhuk, Latent solitons, black strings, black branes, and equations of state in Kaluza-Klein models. Phys. Rev. D 84, 024031 (2011). arXiv:1101.3910 [gr-qc]

22. L.D. Landau, E.M. Lifshitz, The Classical Theory of Fields, Course of Theoretical Physics, vol. 2 (Oxford Pergamon Press, Oxford, 2000)

23. M. Eingorn, A. Zhuk, Weak-field limit of $f(R)$-gravity in three and more spatial dimensions. Phys. Rev. D 84, 024023 (2011). arXiv:1104.1456 [gr-qc]

24. M. Eingorn, A. Zhuk, Asymptotic latent solitons, black strings and black branes in $f(R)$-gravity. Phys. Rev. D 85, 064030 (2012). arXiv:1112.1539 [gr-qc]

25. A.A. Starobinsky, A new type of isotropic cosmological models without singularity. Phys. Lett. B 91, 99-102 (1980)

26. V. Müller, H.J. Schmidt, A.A. Starobinsky, The stability of the deSitter spacetime in fourth order gravity. Phys. Lett. B 202, 198-200 (1988)

27. V.Faraoni, N. Lanahan-Tremblay, Comment on "Solar System constraints to general $f(R)$ gravity”. Phys. Rev. D 77, 108501 (2008). arXiv:0712.3252 [gr-qc]

28. V. Faraoni, Scalar field mass in generalized gravity. Class. Quantum Grav. 26, 145014 (2009). arXiv:0906.1901 [gr-qc]

29. M. Eingorn, J. Novák, A. Zhuk, $f(R)$ gravity: scalar perturbations in the late Universe. Eur. Phys. J. C 74, 3005 (2014). arXiv: 1401.5410 [astro-ph.CO]

30. M. Eingorn, A. Zhuk, Remarks on gravitational interaction in Kaluza-Klein models. Phys. Lett. B 713, 154-159 (2012). arXiv:1201.1756 [gr-qc]

31. A. Chopovsky, M. Eingorn, A. Zhuk, Weak-field limit of KaluzaKlein models with spherical compactification: experimental constraints. Phys. Rev. D 85, 064028 (2012). arXiv:1107.3388 [gr-qc]

32. A. Chopovsky, M. Eingorn, A. Zhuk, Exact and asymptotic black branes with spherical compactification. Phys. Rev. D 86, 024025 (2012). arXiv:1202.2677 [gr-qc]

33. M. Eingorn, S.H. Fakhr, A. Zhuk, Kaluza-Klein models with spherical compactification: observational constraints and possible examples. Class. Quantum Grav. 30, 115004 (2013). arXiv:1209.4501 [gr-qc]

34. A. Chopovsky, M. Eingorn, A. Zhuk, Problematic aspects of Kaluza-Klein excitations in multidimensional models with Einstein internal spaces. Phys. Lett. B 736, 329-332 (2014). arXiv: 1402.1340 [gr-qc]

35. Ö. Akarsu, A. Chopovsky, A. Zhuk, Black branes and black strings in the astrophysical and cosmological context. Phys. Lett. B 778, 190-196 (2018). arXiv:1711.08372 [gr-qc]

36. U. Günther, A. Zhuk, Gravitational excitons from extra dimensions. Phys. Rev. D 56, 6391-6402 (1997). arXiv:gr-qc/9706050

37. N. Arkani-Hamed, S. Dimopoulos, J. March-Russell, Stabilization of sub-millimeter dimensions: the new guise of the hierarchy problem. Phys. Rev. D 63, 064020 (2001). arXiv:hep-th/9809124

38. E. Yalçınkaya, A. Zhuk, Weak-field limit of a Kaluza-Klein model with a non-linear perfect fluid. Grav. Cosmol. 25, 349-353 (2019). arXiv: 1906.08214 [gr-qc]

39. B. Novosyadlyj, V. Pelykh, Y. Shtanov, A. Zhuk, Dark Energy: Observational Evidence and Theoretical Models (Akademperiodyka, 2013)

40. A.A. Starobinsky, Disappearing cosmological constant in $f(R)$ gravity. JETP Lett. 86, 157-163 (2007). arXiv:0706.2041 [astro$\mathrm{ph}]$

41. D.J. Kapner, T.S. Cook, E.G. Adelberger, J.H. Gundlach, B.R. Heckel, C.D. Hoyle, H.E. Swanson, Tests of the gravitational inverse-square law below the dark-energy length scale. Phys. Rev. Lett. 98, 021101 (2007). arXiv:hep-ph/0611184 
42. M. Eingorn, A. Zhuk, Significance of tension for gravitating masses in Kaluza-Klein models. Phys. Lett. B 716, 176-178 (2012). arXiv:1202.4773 [gr-qc]

43. M. Eingorn, A. Zhuk, Hubble flows and gravitational potentials in observable Universe. JCAP 09, 026 (2012). arXiv:1205.2384 [astro-ph.CO]

44. M. Eingorn, A. Kudinova, A. Zhuk, Dynamics of astrophysical objects against the cosmological background. JCAP 04, 010 (2013). arXiv:1211.4045 [astro-ph.CO]
45. Ö. Akarsu, R. Brilenkov, M. Eingorn, V. Shulga, A. Zhuk, Scalar perturbations in cosmological $f(R)$ models: the cosmic screening approach. Eur. Phys. J. C 78, 609 (2018). arXiv:1806.02669 [gr-qc] 